\title{
Run-to-run Variability on Xeon Phi based Cray XC Systems
}

\author{
Sudheer Chunduri \\ Argonne National Laboratory \\ 9700 Cass Avenue, Lemont, IL, 60439 \\ sudheer@anl.gov \\ Vitali Morozov \\ Argonne National Laboratory \\ 9700 Cass Avenue, Lemont, IL, 60439 \\ morozov@anl.gov
}

\author{
Kevin Harms \\ Argonne National Laboratory \\ 9700 Cass Avenue, Lemont, IL, 60439 \\ kharms@anl.gov \\ Samuel Oshin \\ Intel Corporation \\ 2200 Mission College Boulevard, \\ Santa Clara, CA, 95054 \\ samuel.oshin@intel.com \\ Kalyan Kumaran \\ Argonne National Laboratory \\ 9700 Cass Avenue, Lemont, IL, 60439 \\ kumaran@anl.gov
}

\author{
Scott Parker \\ Argonne National Laboratory \\ 9700 Cass Avenue, Lemont, IL, 60439 \\ sparker@anl.gov \\ Naveen Cherukuri \\ Intel Corporation \\ 2200 Mission College Boulevard, \\ Santa Clara, CA, 95054 \\ naveen.cherukuri@intel.com
}

\begin{abstract}
The increasing complexity of HPC systems has introduced new sources of variability, which can contribute to significant differences in run-to-run performance of applications. With components at various levels of the system contributing variability, application developers and system users are now faced with the difficult task of running and tuning their applications in an environment where runto-run performance measurements can vary by as much as a factor of two to three. In this study, we classify, quantify, and present ways to mitigate the sources of run-to-run variability on Cray XC systems with Intel Xeon Phi processors and a dragonfly interconnect. We further demonstrate that the code-tuning performance observed in a variability-mitigating environment correlates with the performance observed in production running conditions.
\end{abstract}

\section{CCS CONCEPTS}

- General and reference $\rightarrow$ Performance; • Networks $\rightarrow$ Network performance analysis; $\bullet$ Hardware $\rightarrow$ Process, voltage and temperature variations;

\section{KEYWORDS}

Variability, performance tuning, OS noise, system noise

\section{ACM Reference format:}

Sudheer Chunduri, Kevin Harms, Scott Parker, Vitali Morozov, Samuel Oshin, Naveen Cherukuri, and Kalyan Kumaran. 2017. Run-to-run Variability on Xeon Phi based Cray XC Systems. In Proceedings of SC17, Denver, CO, USA, November, 2017, 13 pages.

DOI: https://doi.org/10.1145/3126908.3126926

Publication rights licensed to ACM. ACM acknowledges that this contribution was authored or co-authored by an employee, contractor or affiliate of the United States government. As such, the Government retains a nonexclusive, royalty-free right to publish or reproduce this article, or to allow others to do so, for Government purposes only.

SC17, Denver, CO, USA

() 2017 Copyright held by the owner/author(s). Publication rights licensed to ACM. ACM 978-1-4503-5114-0/17/11_..\$15.00

DOI: https://doi.org/10.1145/3126908.3126926

\section{INTRODUCTION}

On many HPC systems, the performance of benchmarks and applications is subject to significant variation when run in the typical production mode [16]. This variability can make HPC systems less productive and performance measurements less reliable in a variety of ways [11]. Systems and applications are often evaluated in terms of their performance characteristics under optimal conditions, and in the presence of variability, systems are less productive than their generally best-case performance numbers would suggest, as any variability would represent a reduction in performance from the optimal value [27].

The ability to measure and report accurate and meaningful performance data is critical to the assessment and improvement of HPC systems, and can become significantly more complicated in environments with variability. Performance data can require multiple executions to collect and cannot be meaningfully reported as a single value but must be expressed as a data distribution. Since opportunities to run at large scale are limited, collecting large data samples can become impractical. Similarly, application tuning becomes more complex, requiring multiple executions to gather statistically significant results and determine whether performance changes are the result of code modifications or the system performance fluctuations [20, 29]. Auto-tuning, an already expensive process, can also become impractically expensive [5]. Further complications brought on by variability include hiding the performance impact of system software changes, complicating the assessment of system health via benchmarks. Variability also makes it difficult to predict job duration and cost, which impacts user productivity and can also complicate job scheduling [3].

This work will examine the nature of performance variability on Cray XC40 systems that utilize the second-generation Intel Xeon $\mathrm{Phi}(\mathrm{KNL})$ processor. Presented are results that quantify the degree of variability that is present on these systems and that identify the various sources that contribute to the cumulative variability observed. Quantification of system baseline variability is important because reported changes in performance can only be evaluated within the context of the underlying level of system variability. It 
is anticipated that a wide variety of benchmark and application performance results will be reported on these systems, and these data can serve to help design and interpret performance studies.

Variability on KNL XC40 systems was studied using a variety of micro-benchmarks, mini-apps, and applications including the Nekbone and MiniFE mini-apps and the LAMMPS and MILC applications. Observed performance variations were over $70 \%$ for some applications and over 2-3X for the benchmarks. Because of the variety of causes of variability in the system, different applications are found to be sensitive to different sources of variability to different degrees. Several sources of variability have been identified, some unique to the Xeon Phi, including variability at the core, tile, node, and network levels. While IO is a known source of variability, it has distinct characteristics and is not tightly coupled to the computeintensive components of an application which are the focus of this study, and therefore will not be included in this work. In addition to identifying sources of variability and quantifying their magnitude, we have identified several mitigation strategies that may be employed to reduce variability when assessing the impact of code changes.

In Section 4.1, we examine the lowest level core and OS noise issues. Section 4.2 considers variability originating at the level of the two core KNL tiles. Section 4.3 examines the causes of variability at the node level as the problem size increases into MCDRAM (multi-channel DRAM) or DRAM. In Section 5, we move away from intra-node variability and examine inter-node variability at the network level. Finally, in Section 6, the entire combination of variability sources and their effects are examined using a set of application codes running up to hundreds of nodes.

\section{RELATED WORK}

Parallel application scalability can be significantly impacted by the node-level performance variability. Several researchers have studied the effects of OS services on performance variability [2, 7]. On multi-core architectures, one approach to concentrate the OS services onto a specific core is through core specialization [21], which effectively isolates the rest of the cores from OS activity. We demonstrate that OS noise can still be significant, especially for micro benchmarking studies; however, we show that its effects on the real applications can be mitigated effectively.

Another potential source of variability is the dynamic frequency scaling effects. Performance variation caused by dynamic overclocking on top supercomputing platforms was demonstrated by Bilge et al. [1]. They noticed performance degradation caused by frequency variation on math kernels and HPC applications. Contention for the shared resources on a node by the co-running codes can lead to performance variability [22]. The shared cache contention can influence the variability between co-located threads on a multi-core processor. As we show in this paper, even the co-located processes can be influenced by cache contention on KNL owing to its tilebased architecture, where two cores on a tile share the same L2 cache.

At the system scale, researchers have identified the network contention as the prime contributor for the variability. Some have used MPI collective benchmarks to demonstrate this; Skinner et al. [24] noticed a 2-3X increase in MPI_Allreduce latency due to network contention from co-located jobs. Hoefler et al. used simulation to study the effect of OS noise on communication operations and concluded that it strongly influences the collective operations [12]. Bhatele et al. [4] and Yang et al. [28] have observed that shared network resource contention is the major contributing factor for variability on dragonfly networks. They have also highlighted that the effects of node placement on the variability were not dominant enough compared to network congestion effects. Jain et al. [15] proposed fabric isolation schemes to address the inter-job effects in the dragonfly networks and have quantified the benefits through simulation.

Tools have been developed to quantify the extent of inter-job effects in HPC systems. For instance, the tool mentioned in [8] measures the effects of inter-job contention by continually running the benchmarks and provides insights using network performance counter data. Several researchers have analyzed the variability on specific systems using real applications. Van Straalen et al. [26] identified the heap management algorithm implementation on Cray $\mathrm{XT}$ as the source of variability observed on AMR applications. A reduced noise kernel was proposed for the Cray XT5 platform and its effect on applications was demonstrated [19]. León et al. [17] categorized the CORAL application benchmarks based on their sensitivity to the network bandwidth and run-to-run variability. Heirman et al. [10] proposed methods to accurately analyze the performance of applications on different architectures considering the variability aspects. In this paper, we not only quantify the extent of variability at all the levels of the Cray XC system hardware, we also present ways to mitigate some of the variability sources.

\section{EXPERIMENTAL SETUP}

In this section, we describe the high-level aspects of our experimental design and the machines used in this study. This overview will describe the broad strategy of the experimental design; more specific details for an experiment are provided in the relevant section.

\subsection{Experimental Design and Measurements}

The investigation began with measuring the performance variability at the lowest level of granularity by running microbenchmarks on a single core with a small number of instructions per iteration. As we captured the variability imparted at this level of the hardware, we applied mitigations to reduce or eliminate the variability and then proceeded to the next-higher level of hardware. At any given stage, it is critical to run each test repeatedly to establish a statistically valid baseline and review the distribution of results.

\subsection{Machines Used}

The majority of the experiments used the Theta supercomputer installed at the Argonne Leadership Computing Facility (ALCF). Theta is a Cray XC40 with 3624 Intel KNL 7230 compute nodes. The compute nodes are connected with the Cray Aries high-speed network in the Cray dragonfly topology. The Intel KNL [25] 7230 has $64 \mathrm{CPU}$ cores with $16 \mathrm{GiB}$ of MCDRAM and $192 \mathrm{GiB}$ of DDR4 2400. The KNL is built based on "tiles" where a tile contains 2 cores and a shared $1 \mathrm{MiB}$ L2 cache. Each core has its own $32 \mathrm{KiB} \mathrm{L} 1$ instruction and $\mathrm{L} 1$ data cache. A core has out-of-order execution and supports four hardware threads with two AVX512 vector units. The 
7230 has a base frequency $1.3 \mathrm{GHz}$, and AVX frequency of $1.1 \mathrm{GHz}$ when the instruction stream contains approximately more than $40 \%$ of AVX instructions. The Intel Turbo feature can dynamically scale the frequency up to $1.5 \mathrm{GHz}$ for a single tile or $1.4 \mathrm{GHz}$ for several tiles. Individual cores cannot scale their frequency up or down. The KNL MCDRAM can be configured on boot into one of several combinations of memory and cluster modes [25]. The two memory modes we focus on in this paper are the cache and flat modes, and we use the quadrant cluster mode. The cache memory mode configures the MCDRAM as a last level direct-mapped cache of the DRAM. The flat memory mode configures the MCDRAM as a separately addressable memory space with its NUMA characteristic as "far" such that the OS will not allocate into it, leaving the MCDRAM for the application. The quadrant cluster mode defines how the tag directory map of the DRAM and MCDRAM is handled. We selected quadrant mode for the best performance versus ease-of-use tradeoff.

The Aries interconnect is a three-level dragonfly topology network. The first two levels (rank-1 and rank-2) are copper based with $10.5 \mathrm{~GB} / \mathrm{s}$ bi-directional bandwidth per link, and the rank-3 level is optical with $9.38 \mathrm{~GB} / \mathrm{s}$ per link. Each node has a peak injection rate of $16 \mathrm{~GB} / \mathrm{s}$.

In addition to evaluating the tests on Theta, we leveraged two systems from the National Energy Research Scientific Computing Center (NERSC), Cori and Edison. Cori is a Cray XC40 system similar to Theta but much larger consisting of 2004 Intel Haswell E5-2698 v3 compute nodes and 9688 Intel KNL 7250 compute nodes. The Cori KNL nodes are 68-core parts with an increased clock rate of $100 \mathrm{MHz}$ over the Theta KNL nodes. In this study, we use only the KNL nodes. Cori also uses the Aries high-speed interconnect similar to that described above for Theta. NERSC's Edison machine is a Cray XC30 system. Edison uses Intel Ivy Bridge processors that vary significantly from a KNL, but we do not describe them in detail here. We use Edison only to confirm the network-based experiments. Edison uses the same high-speed Aries interconnect with a 3-level dragonfly topology. The Aries networks on Edison and Cori are larger than that of Theta; hence they are prone to potentially more frequent occurrences of high network congestion rate.

\section{INTRA-NODE VARIABILITY}

Within this section, various sources of intra-node variability will be examined and characterized using a set of benchmarks. For the core level studies, we run all experiments on a given node looking at only the run-to-run variation rather than variation between different nodes. However, we have repeated the experiments on multiple nodes to validate the statistical significance of the observations. All test cases in this section are performed on Theta. Where possible, mitigation strategies are recommended to reduce the overall variability resulting from a given source. These strategies are applied as the test cases are built up to help isolate new sources of variability.

We used the following microbenchmarks: Selfish [2], FWQ [12], HACCmk [6], STREAM [18], and Naïve Matrix Multiplication (NMM). NMM is a simple double-precision square matrix multiplication algorithm.

\subsection{Core-Level Variability}

At the core level, a well-known and primary cause of variability can be the activity from the OS [2]. The Selfish [2] benchmark is used to estimate OS noise by running an idle timed loop and measuring time detour from the expected threshold. Figure 1 shows the OS noise (detour) of up to $250 \mu$ s on a randomly picked core running the benchmark. This level of noise can have a significant impact on kernels when examining performance at small time scales. The Cray system provides a feature named core specialization, which affinitizes the OS activities to specific cores/hardware threads. The user can specify the number of resources to reserve, and the OS threads will be bound starting with the highest core number. Figure 2 shows 10X reduction of noise to about $25 \mu$ s when core specialization (reserving one core for OS) is used. This noise can be observed within an application kernel as well. Here, we use a naïve matrix multiplication (NMM) with no optimizations and the number of elements sized to fit entirely in the L1 cache. In Figure 3, we show the effects of the OS noise on this kernel. The application processes running on core 0 and core 1 are executing much more slowly than the other processes owing to contention with the OS as discussed above. The results of the same experiment with core specialization are shown in Figure 4. By confining the OS threads to the last core, the absolute performance difference between the processes is greatly reduced.

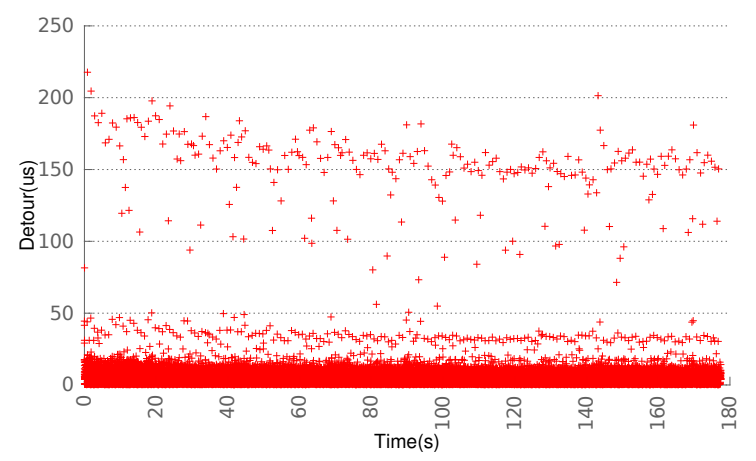

Figure 1: OS noise effects on a core by default

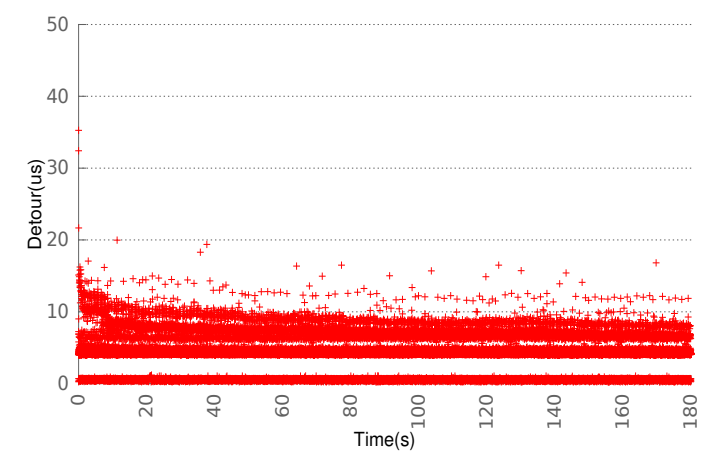

Figure 2: OS noise effects on a core with OS core affinity (core specialization)

Prior studies [1,23] showed performance variability occurring due to turbo mode on Xeons, so we examined it as a possible cause of variability on our systems. Our experiments on the KNL node did not show any material difference from turbo mode for a single node. Variability from frequency scaling is about $0.5 \%$ with inconclusive 


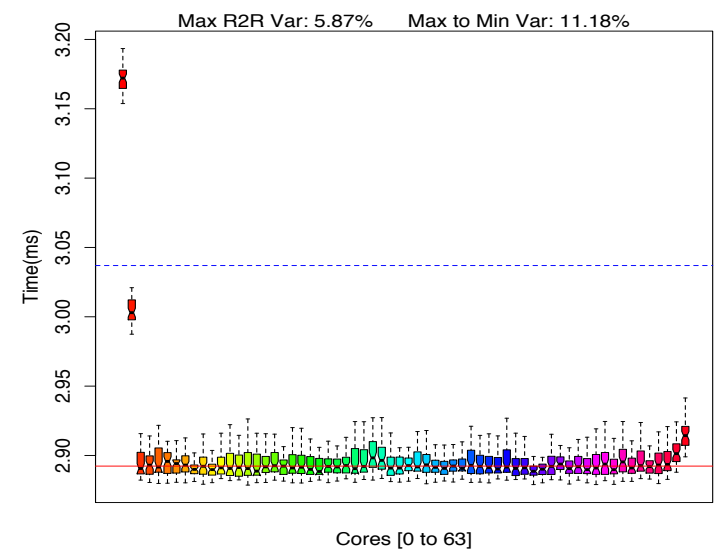

Figure 3: OS noise effects on matrix multiply kernel by default

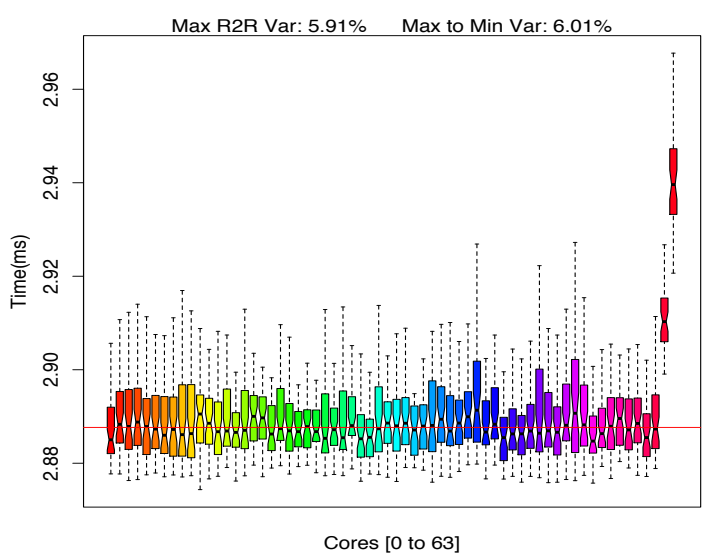

Figure 4: OS noise effects on matrix multiply kernel with OS core affinity (core specialization)

trends; variability can go up or down by $0.5 \%$ when turbo is enabled. However, turbo mode can improve performance by $1 \%$ to $7 \%$ for the benchmarks we evaluated.

The impact of core-level variability on applications has been evaluated using three codes. HACCmk [6] is the major compute kernel from the HACC code, FWQ [12] is a synthetic compute benchmark that repeatedly performs a fixed amount of work, and again we use our matrix multiply test. Each code was configured to use data from only the $\mathrm{L} 1$ cache and run for differing lengths of time ranging from 1 millisecond to 1 second, and the range of variability was observed. As Figure 5 shows, the observed variability between the runs decreases as the run duration increases, with core-level variabilty approaching zero as the run time approaches 1 second. This reduction in variabilty can be explained by an averaging out of the core-level variability over sufficiently long intervals.

Some variability is still observed at the core level, and the exact cause continues to be investigated. Architectural features of the KNL many-core node can potentially induce core-to-core variability. As an example, core proximity to the memory controller may result in asymmetric memory latency or on-die mesh bandwidth. In multinode systems, variability between cores across different nodes could exist due to manufacturing differences, although frequency binning

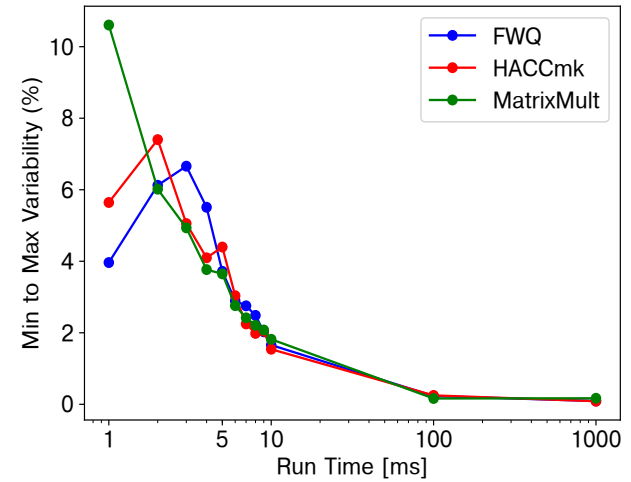

Figure 5: Performance variance vs. runtime of the benchmark.

during chip production can significantly reduce variability from this source.

Recommendation: Use OS features such as core specialization that reduce the impact of the $O S$ on the application. The impact of core-level variability effects can be minimized by using sufficiently large run times.

\subsection{Tile-Level Variability}

The KNL features a tile architecture with two cores sharing the L2 cache. The impact of the shared L2 cache is illustrated using the weak-scaled MPI version of HACCmk. The variability when a serial version of HACCmk is run on both cores on the tiles is shown in Figure 6. (The cores from first and last tiles are excluded for this experiment in order to avoid the OS noise effects possible on these tiles even with core specialization.) The maximum of the run-to-run variability range across all the 60 cores is $5.3 \%$, and the maximum overall core-to-core variability range is $12.4 \%$. A bimodal runtime distribution can be seen in the figure. This bimodal time distribution correlated well with the $\mathrm{L} 2$ cache miss count distribution shown in Figure 7. Around 2X variability in the L2 cache miss count is observed.

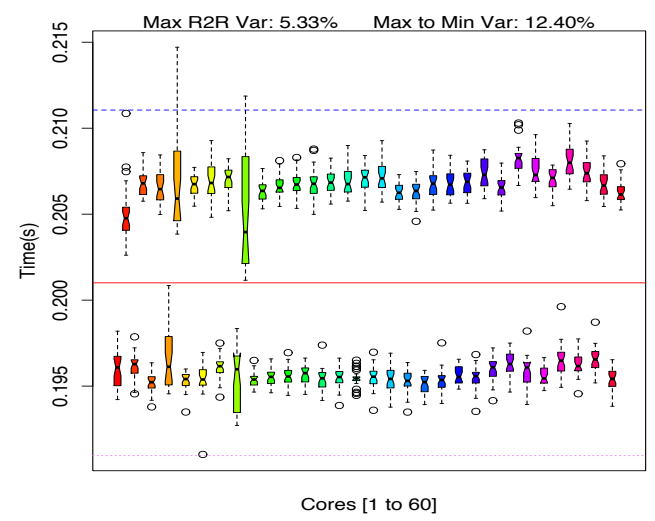

Figure 6: HACCmk runtime variability using two cores per tile

The effects of variability for this kernel can be mitigated when only one core per tile is used. As shown in Figure 8, with one core per tile, the overall core-to-core variability range is only $2 \%$.

This experiment was repeated across several nodes to validate the statistical significance of the observations made here. The average 


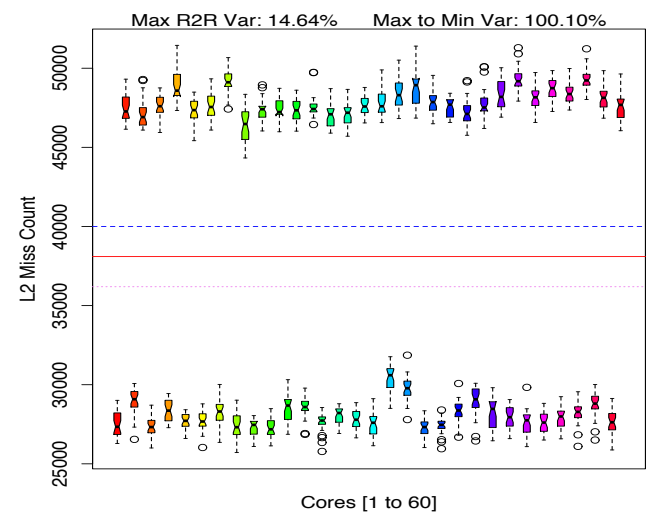

Figure 7: HACCmk L2 miss count variability using two cores per tile

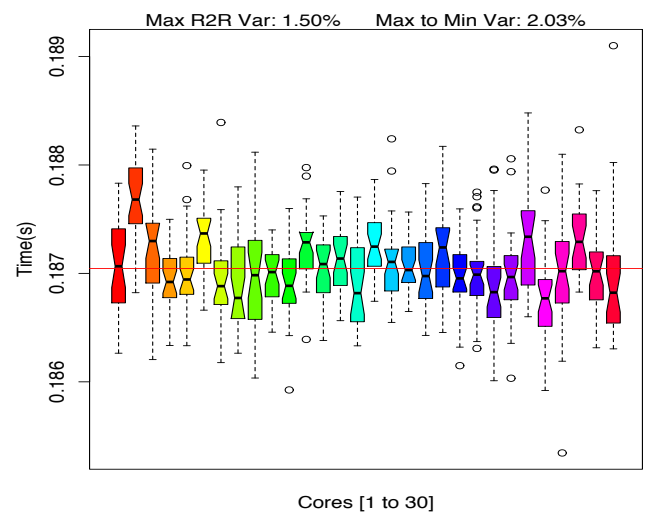

Figure 8: HACCmk runtime variability using one core per tile

variability observed across these nodes is $4.97 \%$ when using one core/tile and $14 \%$ when using two cores/tile.

NMM is used to study the variability as a function of memory footprint size. Similar to the earlier experiment using HACCmk, NMM is run on 60 cores on a node with different matrix sizes such that the working set size varies from $20 \%$ to $200 \%$ of the L2 cache capacity. Figure 9 shows the percentage of maximum to minimum core-to-core variability when two cores and one core per tile are used. Starting at about $80 \%$ of L2 capacity, cache conflicts resulted in higher variability with two processes/tile. Variability for memory footprint in the [ $80 \%$ of L2 - $200 \%$ of L2] range is subject to application address distribution and access patterns when two cores/tile are used. Lower variability is observed when only one core per tile is used.

As the memory footprint approaches L2 cache size, cache contention (as indicated by the L2 cache miss count) between the two cores resulted in the performance variability. As of now, we do not understand the root cause for the cache-sharing unfairness. ${ }^{1}$ However, using one core per tile mitigates this contention and greatly reduces the overall variability.

\footnotetext{
${ }^{1}$ The tile-level variability is not observed on other KNL clusters and also it is not observed on Theta after sofwatre updates, hence, we reason out that the variability could be an artifact of the software and not inherent in the hardware.
}

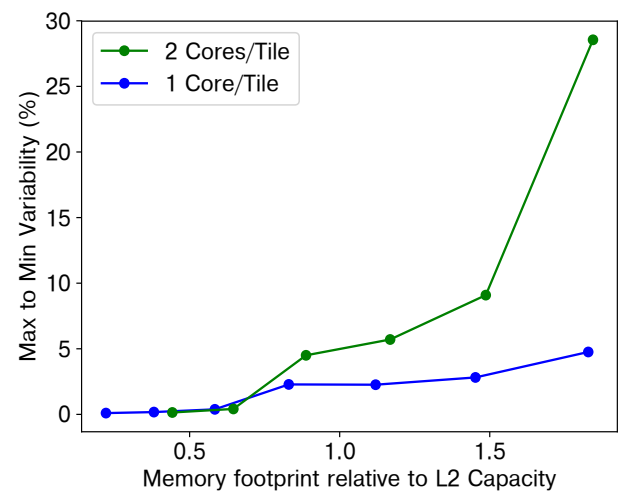

Figure 9: Impact of L2 cache sharing vs. runtime variability

Recommendation: Characterize any core-level shared resources such as cache to determine possible fairness issues when contention occurs.

\subsection{Memory Mode Variability}

One of the unique features of the KNL processor is the two types of memories it has: DRAM and MCDRAM. As mentioned in Section 3.2, the KNL can be configured in different modes. In this section, the differences between the cache and flat mode in terms of the variability are characterized. The peak STREAM Triad bandwidth for DRAM is $90 \mathrm{GiB} / \mathrm{s}$, and MCDRAM is over $480 \mathrm{GiB} / \mathrm{s}$.

In flat mode, the MCDRAM is a separately addressable memory and has the properties very similar to those of DRAM except for higher available bandwidth. When MCDRAM is in cache mode, it is a direct mapped cache. Multiple memory locations from DRAM can map to a single location in the MCDRAM cache. The mapping uses the physical addresses, and not the application-level logical addresses. Even when an address is contiguous in the logical address space, the physical addresses that the OS assigns to the application memory are not required to be contiguous. This treatment can cause conflicts in the MCDRAM cache when a portion of the MCDRAM cache is used, thus effectively resulting in reduced memory bandwidth. This reduction can vary from run to run, as OS page allocation may change from run to run. We characterize the effect of this by using the STREAM Triad benchmark.

The benchmark is run on multiple nodes repeatedly for 10 times, and the variability in the measured bandwidth between nodes is captured. A working set size of 7.5 GB, which is less than half of the available MCDRAM memory is used. The Triad bandwidth measured on 50 different nodes in flat MCDRAM-only mode is around $485 \mathrm{~GB} / \mathrm{s}$, and it is consistent across the nodes with less than $1 \%$ node-to-node variability. Figure 10 shows the bandwidth for the same working set on 50 nodes in MCDRAM cache mode. The peak bandwidth achieved in the cache mode is just around $345 \mathrm{~GB} / \mathrm{s}$. However, several nodes achieve lower than this peak, with a specific node achieving just around $170 \mathrm{~GB} / \mathrm{s}$. The node-to-node variability in the effective bandwidth measured is around $2 \mathrm{X}$. This kind of variability can make even a perfectly balanced code run appear imbalanced, which means time lost to hardware inefficiencies that are potentially unknown and cannot be controlled by the user.

Node performance monitoring event counters can be used to understand the reasons for the high node-to-node variability in the 


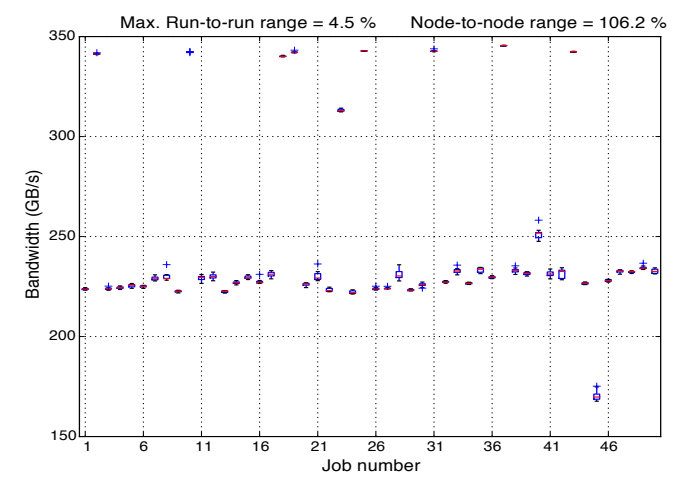

Figure 10: Cache Quad (CQ) mode node-to-node bandwidth for the STREAM benchmark with working set size $7.5 \mathrm{~GB}$

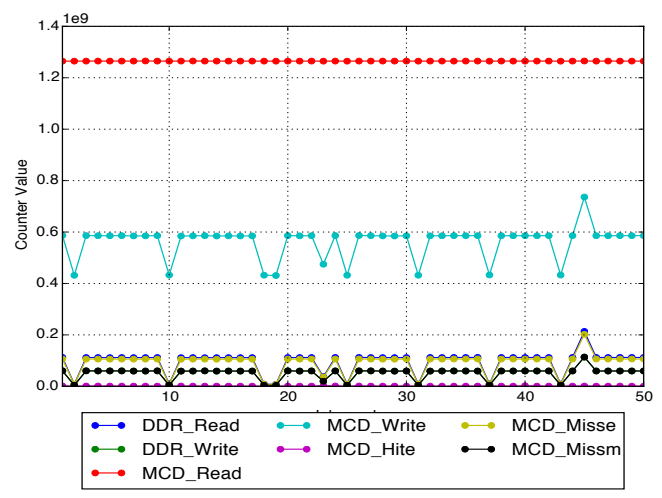

Figure 11: Cache Quad (CQ) mode node-to-node uncore events for the STREAM benchmark with working set size 7.5 GB

cache mode. The number of reads and writes for both MCDRAM and DRAM can be counted using these events [13]. Additionally, in the cache mode, MCDRAM hits and misses when MCDRAM is in either a clean or dirty state can also be measured. The summation of MCD_Misse and MCD_Missm (MCDRAM misses when MCDRAM is clean and dirty, respectively) represent the number of writes from L2 to MCDRAM. Figure 11 shows the uncore counter data corresponding to one run of the STREAM benchmark run in the cache mode. The node that has a lower bandwidth in Figure 10 has a correspondingly higher MCDRAM miss ratio (a higher number of MCDRAM writes) in Figure 11, thus indicating the potential conflicts incurred in the MCDRAM cache. The untile counter data for the Flat MCDRAM-only mode has the same miss ratio across all of the nodes as shown in Figure 12.

The above observations are for a specific working set size (7.5GB); we then verified cache mode variability for other sizes that fit within MCDRAM. Figure 13 shows the node-to-node variability across 50 nodes with MCDRAM as a cache for different working set sizes that are smaller than the MCDRAM capacity. Bandwidth on some of the nodes is low with all the working set sizes. Since the workaround to address the page conflict issue in cache mode is unknown; therefore, running in the flat quad mode is the recommended mitigation. Cray's Zone sort [14] algorithm, enabled by default on Theta and Cori, sorts the MCDRAM free page list with each job invocation. The expectation is that this sort would help address the MCDRAM page conflict issues; however, the state of the free page list could

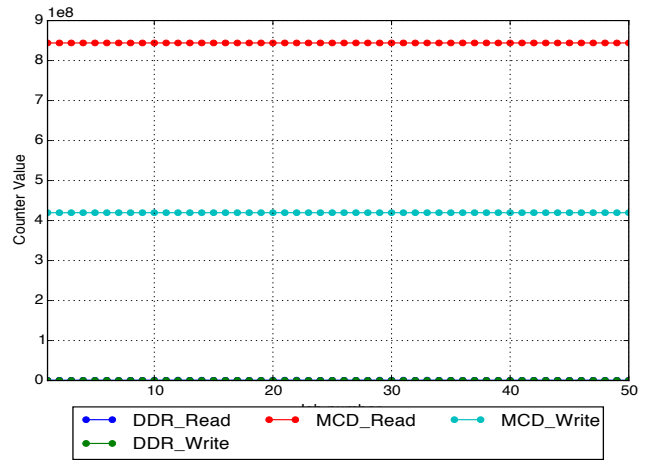

Figure 12: Flat Quad (FQ) mode node-to-node uncore events for the STREAM benchmark with working set size $7.5 \mathrm{~GB}$

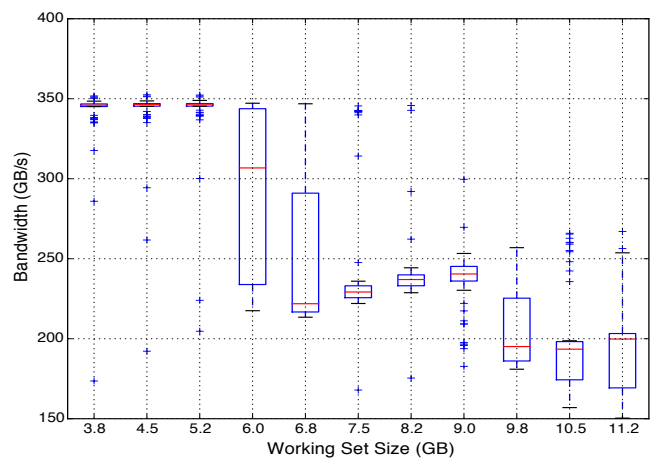

Figure 13: Cache Quad (CQ) mode node-to-node bandwidth variability across 50 nodes for the STREAM benchmark with different working set sizes

potentially be different from node to node depending on the prior application that used the MCDRAM on that node.

We demonstrate the effects of memory mode variability on the MiniFE [6] application. MiniFE is a memory bandwidth-intensive application; we study its performance variability in the cache mode. We run MiniFE with different problem sizes and report the performance in GFlops. It is run on 128 single-node jobs in cache quad mode on Cori, and the mean performance across the 128 jobs is noted. Figure 14 shows the mean performance along with the standard deviation (as error bars). The variability is high when the problem size fits within the MCDRAM (16 GiB), and low when the problem size fits only in the DRAM. While Zone sort helps in reducing the variability range across the 128 jobs, it does not help reduce the variability entirely. MiniFE running in node MCDRAM flat memory mode showed no variability.

Recommendation: Shared node-level resources can have a signficiant impact on variability and need to be characterized to understand the performance. Memory bandwidthintensive applications could be run in flat mode for repeatable performance.

\section{SYSTEM-LEVEL VARIABILITY STUDY}

The scale of the interconnection networks is increasing along with scale of the supercomputers. While the flop-rate is expected to grow, the challenge for application performance is the nominal 


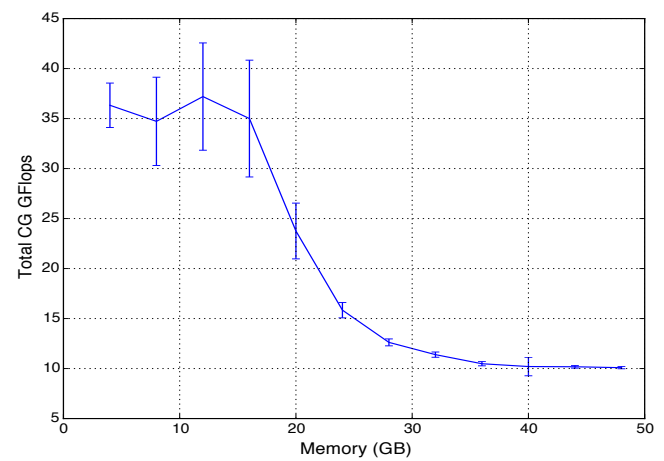

Figure 14: Node-to-node variability of MiniFE across 128 nodes of Cori with increasing problem size [Zone Sort $\mathrm{ON}$ ]

network performance growth. Higher-radix, lower-diameter network topologies such as dragonfly are widely used in the recent HPC systems. As the network scale increases, the network diameter increases and the bisection bandwidth relative to the number of nodes decreases. Resource managers typically attempt to maximize job throughput and system utilization. Consequently, multiple application jobs running on the system share the network resources such as routers and links, and this resource sharing can potentially lead to great performance variability from run to run.

We first quantify the extent of this variability using MPI collective benchmarks. While designing the experiments in this section, the recommendations described earlier for reducing the core- and node-level variability are adhered to.

\subsection{MPI Collective Benchmarks}

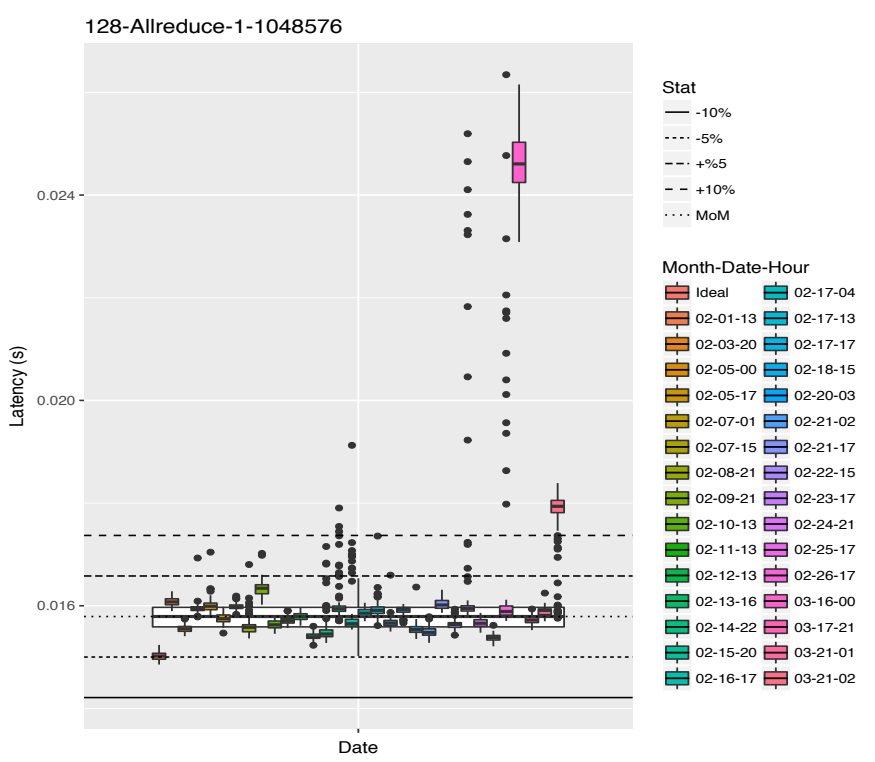

Figure 15: 128 processes (one process per node) MPI Allreduce Latency with a message of $1 \mathrm{M}$ DOUBLE data

The MPI collective benchmarks are run on different node sizes on Theta using the flat memory mode. The benchmarking job is run on several days, which potentially results in a randomized job placement and job mix. We have run the benchmark job in an isolated environment where no other job is running on the system. We refer to this as the ideal case. Figure 15 shows the latency for the MPI_Allreduce with a message size of $8 \mathrm{MB}$ (1M doubles) on 128 processes (using 1 process per node) on Theta. We use only 1 process per node in order to avoid any source of variability coming from the node level. Within each job, the collective call is repeatedly run 100 times; each box in the figure represents the variability across the 100 runs. The run-to-run variability for different days is shown with a corresponding box. The bottom and top of the box are the $25^{\text {th }}$ and $75^{\text {th }}$ percentile respectively, and the band near the middle of the box is the $50^{\text {th }}$ percentile, or the median. The middle horizantal line shows the median of medians, and the other four horizontal lines show $+/-5 \%$ and $+/-10 \%$ difference from the median of medians.

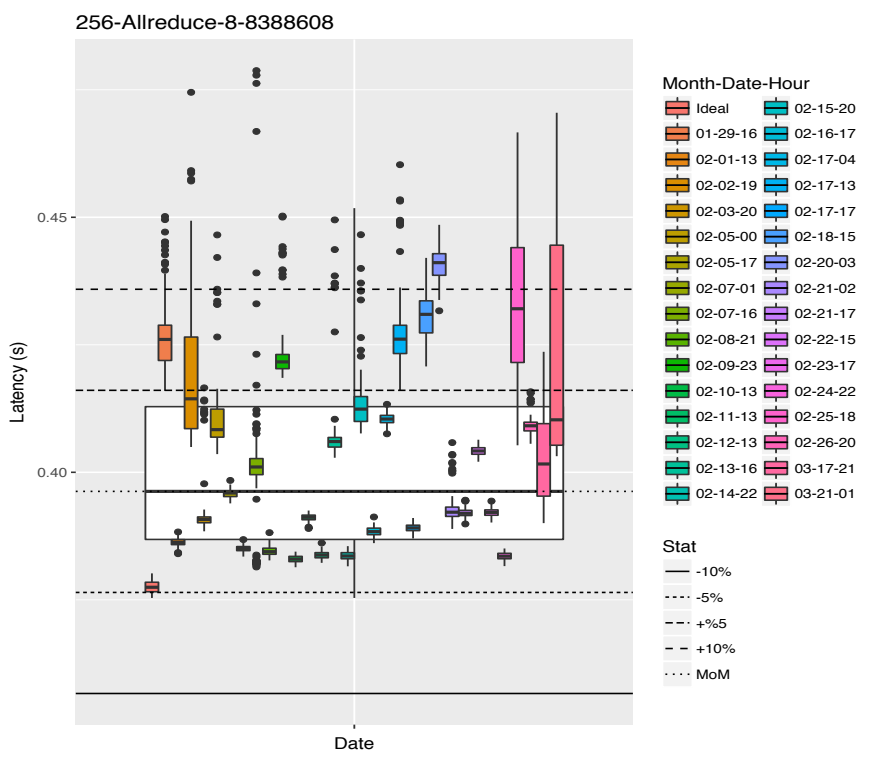

Figure 16: $2 \mathrm{~K}$ processes (8 processes per node on 256 nodes) MPI Allreduce Latency with a message of $8 \mathrm{M}$ DOUBLE data

The run in the isolated system has the lowest latency of around $15 \mathrm{~ms}$, and the benchmark has good repeatability irrespective of the node placement used (not shown in the figure). The median latency across all the days (median of medians) is $5 \%$ higher than this level. The median latency on two specific days is around $10 \%$ and $50 \%$ greater, respectively, than the overall median latency. For some days, while the median latency is well within $5 \%$ from the overall median, there are a few outliers that are around $50 \%$ higher than the overall median. As only one process per node was used in this experiment, the variability observed here is attributable solely to the network congestion.

We now experiment with a configuration that has higher nodelevel parallelism. The MPI_Allreduce variability for $2 \mathrm{~K}$ processes using 8 processes each from 256 nodes with $64 \mathrm{MB}$ (8M doubles) message size is shown in Figure 16. The MPI processes within a node are mapped such that no two MPI processes are mapped onto the cores on the same tile to avoid the effects of L2 contention as discussed in Section 4.2. While the run-to-run variability on most of the days is small, there are a few cases where it is as high as $15 \%$. The higher latency can be attributed to the higher inter-job contention during the job run. 


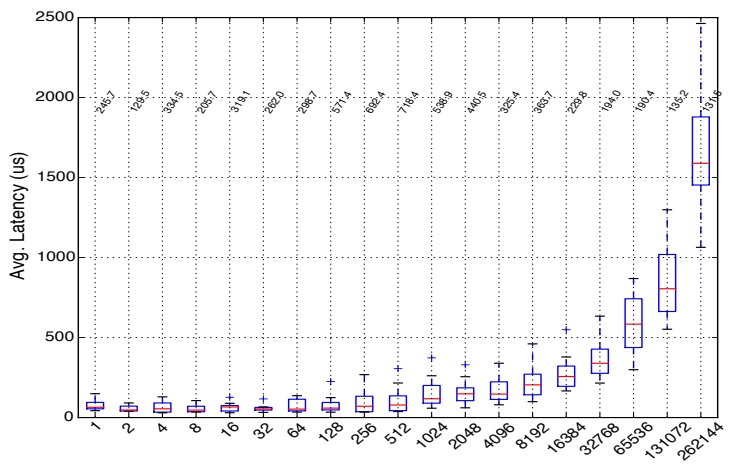

Figure 17: 256 processes (256 nodes - 1 ppn) on Edison MPI Allreduce Latency with different message sizes

We now show the latency of MPI_Allreduce with different message sizes on a 256-node allocation on Edison where the benchmark job is repeated 10 times. Figure 17 shows variability across these 10 different jobs. Edison also uses the dragonfly topology, and we wish to quantify here the effects of inter-job contention on Edison. For the smaller message sizes, which are latency sensitive, the jobto-job variability is as high as 7X (the slanted numbers within the plot represent the percentage of maximum to minimum variability). This study on Edison brings forth the point that the variability is inherent for the Cray's dragonfly implementation and is independent of the node architecture.

We then studied the routing mode and reordering effects on the variability of MPI_Allreduce latency. The routing mode decides the routing policy for the message on the dragonfly topology. The choice of routing mode used is user configurable. The job scheduler provides two different heuristic-based reordering methods for remapping MPI processes onto nodes. Figure 18 show the variability for MPI_Allreduce with a message of 256 Double precision data under different routing modes and reordering methods. The variability across the runs within a job and the variability across the 4 different jobs is shown in the figure. While the routing modes such as MIN-HASH and IN-ORDER, which are static routing modes, enable relatively low average latency compared to other modes, the overall variability is still significant. However, using the static routing modes can help mitigate the variability that occurs due to adaptive routing. At the moment, for mitigating the network variability, we do not yet have a recommended strategy other than running in an isolated system.

\section{APPLICATIONS}

Now that we have quantified the variability at the node level and the system level using the benchmarks as described in the earlier sections, we study how applications are affected by variability sources and how we can perform performance optimization in the presence of this variability. We study the applications MILC [6], Nekbone [6], and LAMMPS [6], which have different computation and communication characteristics, and analyze how those applications are impacted by the variability sources identified in the earlier sections.

The applications and input configurations have been selected to obviate the need for any significant $\mathrm{I} / \mathrm{O}$, and we measure only

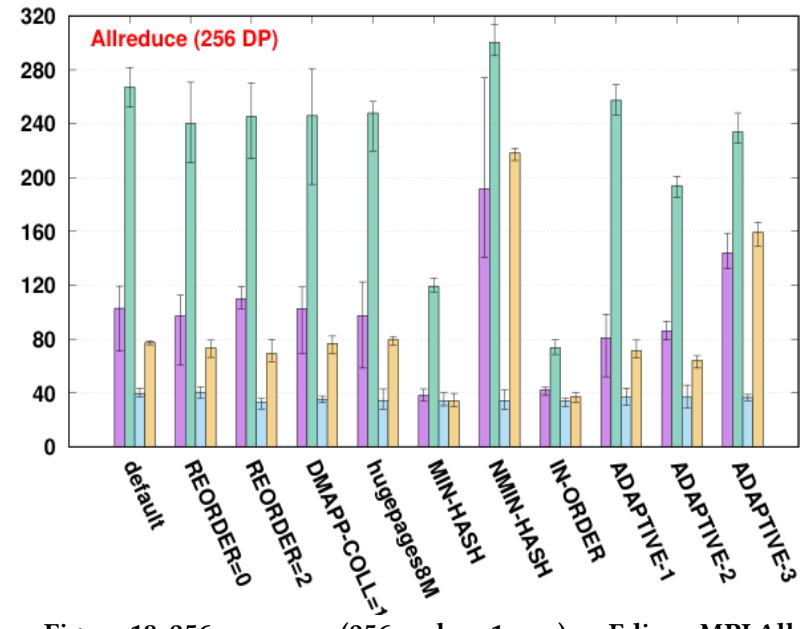

Figure 18: 256 processes (256 nodes - 1 ppn) on Edison MPI Allreduce Latency for $1 \mathrm{~K}$ message with different routing modes

the computational portion of the application. First, we study the application running under a production configuration to obtain a baseline profile for the application. We will then run the application under a "reduced variability" configuration with both the initial code and the tuned code. We strive to demonstrate that with a low-variability environment, performance studies do not require a significant number of iterations to obtain a statistically significant average performance. We demonstrate that the performance gain obtained in the low-variability environment will translate into the normal environment by taking large samples for both configurations and showing that the average performance has improved.

\subsection{MILC}

MILC is a suite of applications for simulating quantum chromodynamics (QCD). The specific application used is the su3_rhmd_hisq with a lattice size of $96^{3} \times 48$ using 8192 MPI processes on 128 nodes. This configuration results in $24^{4}$ lattice sites per node, which results in a memory usage that fits within the $16 \mathrm{GiB}$ of MCDRAM. Table 1 shows the performance profiling when run in the production configuration under the MCDRAM cache mode. The performance difference between a slow run and a fast run is 371.8 seconds or approximately $19 \%$ of the total runtime. From the profile, we see that the majority of that difference, about 273 seconds, is from MPI.

\begin{tabular}{|c|c|c|c|c|}
\hline Run & \multicolumn{2}{|c|}{ Total } & \multicolumn{2}{c|}{ MPI } \\
& Percentage & Time (s) & Percentage & Time (s) \\
\hline Fast & $100 \%$ & 1892.39 & $35.9 \%$ & 678.73 \\
Slow & $100 \%$ & 2264.21 & $42.0 \%$ & 951.75 \\
\hline
\end{tabular}

Table 1: MILC profiling results in production configuration

Although we do not have a precise mitigation for variability induced by the application beyond running it in isolation, instead MILC is run in flat mode, which happens to have a small number of nodes (500) reserved on Theta in sequential order. Running under this configuration provided the minimum variabilty. The su3_rhmd_hisq application provides a detailed set of output for the various solver steps, which can be processed by a script to generate a performance result in MFLOP/s. The FLOPs in this case are as defined by the MILC application developers. We run three tests 
which exhibit less that $1 \%$ variability and achieve a performance of 44,839.56 MFLOP/s. A simple optimization for MILC is to apply an optimized rank-to-node mapping which can be accomplished with Cray's grid_order tool. Running the MILC application with this modified MPI rank mapping achieves 54,821.88 MFLOP/s resulting in $22.2 \%$ performance gain. Figure 19 shows the results for running this configuration under cache mode both with and without the job placement optimization. A total of 20 tests were run for each base case and optimized case, where we find the one-sample t-test for both sets is $2.2 \mathrm{e}-16$. The mean performance for the base case is $36,450.22 \mathrm{MFLOP} / \mathrm{s}$, and the mean performance of the optimized case is $44,954.79 \mathrm{MFLOP} / \mathrm{s}$, for a similar improvement of $23.3 \%$. As can be seen in Figure 19, the ranges for the absolute performance overlap. If only a single random sample was used, a user could be led to believe that the original configuration had better performance than the optimized version.

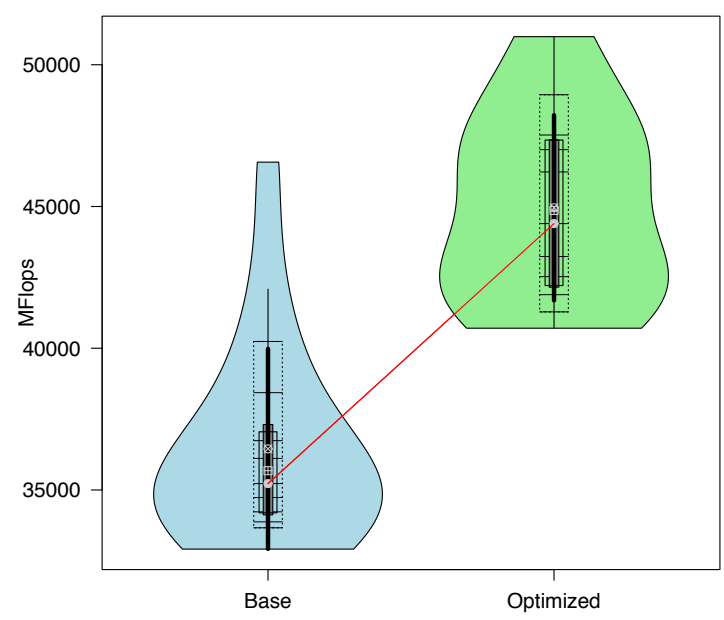

Figure 19: MILC performance comparison of the base case and the optimized case under the production environment

\subsection{Nekbone}

The Nekbone application is a mini-app for the CFD code, NEK5000. Nekbone solves the 3D Poisson equations using an iterative conjugate gradient method. Nekbone has a number of fine-grained timers which measure the three major subcomponents: memory bandwidth-bound dot product type (DAXPY), compute-bound matrix multiplication type (MXM) compute components, and the collective communication (COMM) component. We analyze here how these components are affected by different sources of variability.

Nekbone is set up with a ploynomial order of 16 and 512 elements per process, and 63 processes on a single node of Theta are used. With this input configuration, Nekbone is memory bandwidth bound. We repeatedly run the application 10 times within a node, and the job is run on 70 nodes. Figure 20 provides the time breakdown for the three different kernel components of Nekbone within one run in each of 70 jobs under the production environment. The variability observed in the total time correlates closely with the DAXPY type subcomponent. The DAXPY type subcomponent is a memory bandwidth-sensitive computation, and hence it is affected by the cache mode effects in a manner similar to what is presented with the STREAM benchmark in Section 4.3. Whereas the range of variability with the STREAM benchmark is around $100 \%$, the variability with Nekbone is measured to be around $21.95 \%$. Given that the primary component that dominated variability is the DAXPY kernel, we can run this test under flat mode to reduce the variability. Figure 21 provides the time breakdown for Nekbone within one run on 70 nodes in flat mode. A reasonably low variability level of $3.57 \%$ is observed across the jobs on different nodes.

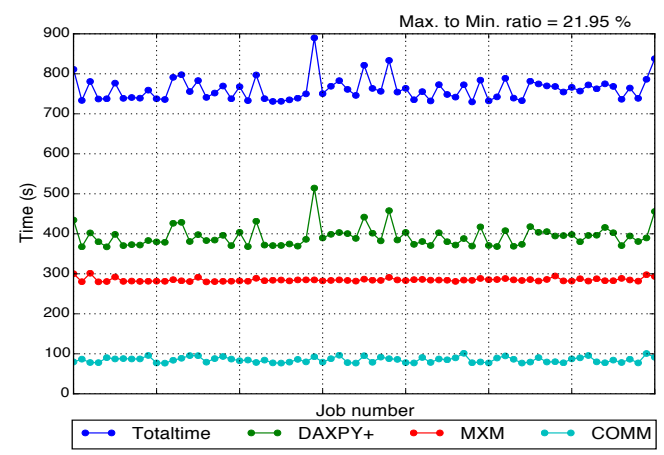

Figure 20: Node-to-node variability for Nekbone: 512 elements, 16 polynomial order on nodes of Theta in cache-quad mode

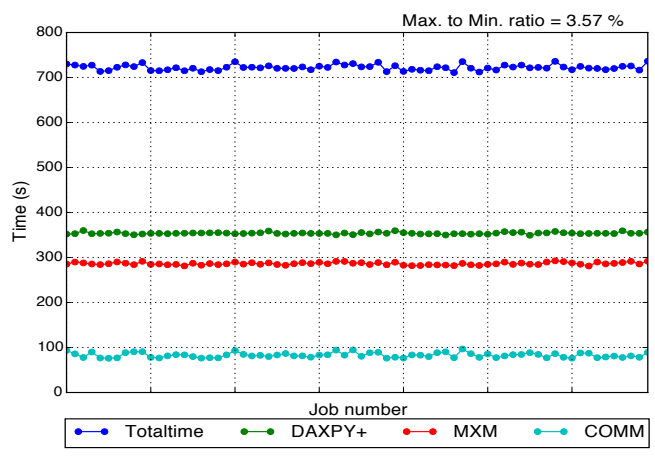

Figure 21: Node-to-node variability for Nekbone: 512 elements, 16 polynomial order on nodes of Theta in flat-quad mode

To further qualify our findings detailed above, we can examine the variability of the total solve time within the node and across the nodes. Figure 22 shows the run-to-run variability range (defined here as the difference between maximum and minimum time) across 10 runs within a job and the variability from node to node across the 70 different nodes that are in flat-quad mode. The maximum run-to-run variability observed was $2.68 \%$, and the node-to-node variability was $4.91 \%$. The same application when run on cachequad nodes leads to higher variability ranges with a maximum run-to-run variability of $18.81 \%$ and a node-to-node variability of $23.34 \%$ as shown in Figure 23.

Nekbone performance can be optimized by applying a modification that improves the performance of small matrix multiplication. Intel has contributed work on the libxsmm project [9] for accelerating small matrix multiplication on the KNL processor. We build Nekbone with the libxsmm library, replacing the Nekbone native matrix multiply kernels. This substitution provides an improvement of $20.7 \%$ over the base case when running under the reduced varibility environment. Figure 24 demonstrates the results that 


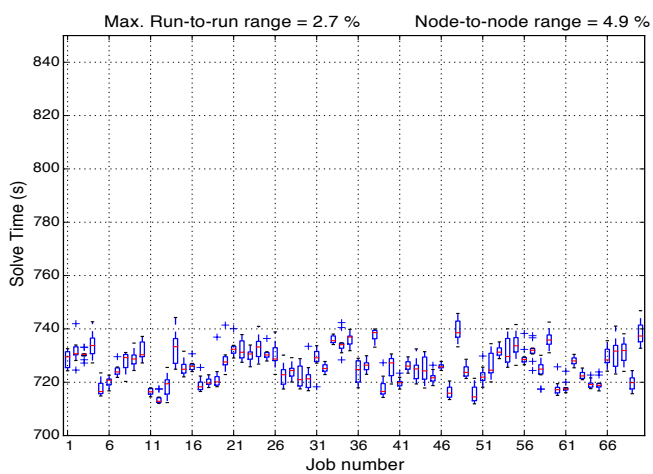

Figure 22: Node-to-node variability for Nekbone: 512 elements, 16 polynomial order on nodes of Theta in flat-quad mode

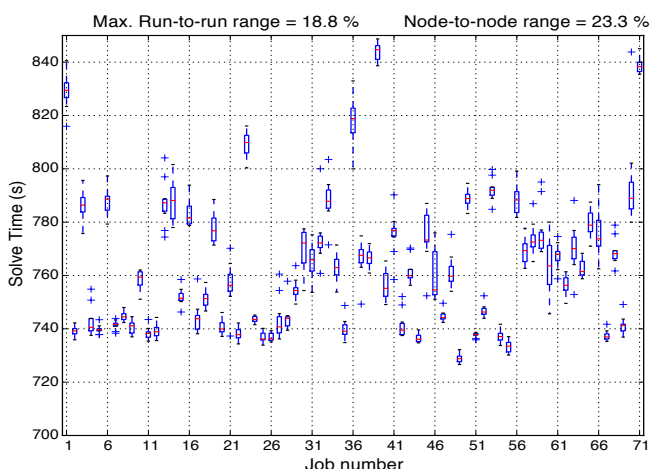

Figure 23: Node-to-node variability for Nekbone: $\mathbf{5 1 2}$ elements, 16 polynomial order on nodes of Theta in cache-quad mode

are achieved when running in the production environment (cache mode) for the base and optimized cases. We see that the mean performance improvement is $18.8 \%$ and that the $95 \%$ confidence interval for improvement is between $18.5 \%$ and $19.0 \%$. Again, in Figure 24, if any two random samples were selected, the application could show a performance change of between $2 \%$ and $35 \%$.

\subsection{LAMMPS}

LAMMPS is a classical molecular dynamics code; it runs on single processors or in parallel using message-passing techniques and a spatial-decomposition of the simulation domain. We run the LAMMPS using the RHODO benchmark on a single KNL node on Theta. Table 2 reports the variability from run to run with different run configurations, with the default configuration using all 64 cores with 2-way multi-threading. The variability ranges for this case in cache mode and flat mode are $14 \%$ and $13.2 \%$, respectively. Using just one thread per core reduced the variability to $4.9 \%$, thus indicating that using two threads per core potentially leads to L2 cache conflicts due to preferential L2 treatment between the two cores on a tile. Using core specialization and only 63 cores further reduced the variability to $2.9 \%$.

With a reduced variability environment specified, we can evaluate the performance of using the native versus optimized math libraries. The base case is run under the low-variability environment with a computational loop requiring 1247.3 seconds. LAMMPS is rebuilt linking with the Intel MKL and run under the low-variability

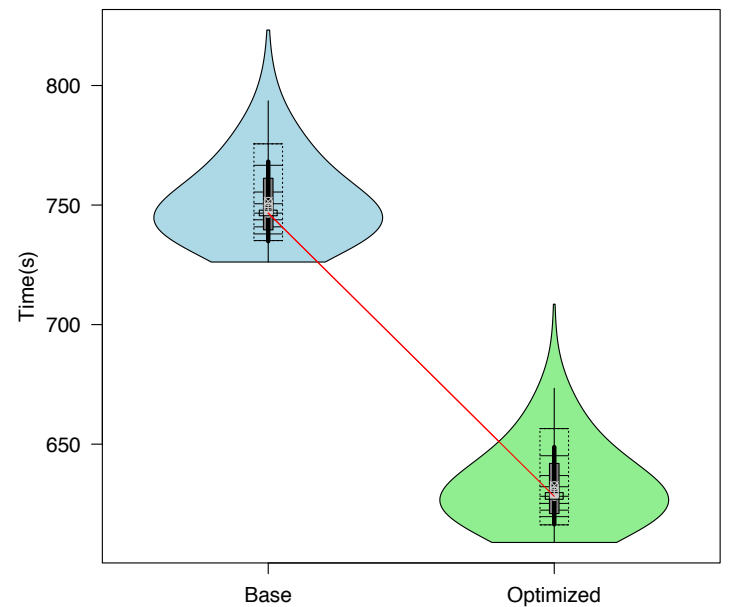

Figure 24: Nekbone solve time comparison of the base case and the optimized case under the production environment

\begin{tabular}{|l|l|}
\hline Run Configuration & Variability \\
\hline 64 cores 2 OMP CQ & $14 \%$ \\
64 cores 2 OMP FQ & $13.2 \%$ \\
64 cores 1 OMP CQ & $4.9 \%$ \\
63 cores 1 OMP CQ (core spec) & $2.9 \%$ \\
\hline
\end{tabular}

Table 2: LAMMPS - percentage of variability with different run configurations

environment with a loop time of 617.7 seconds for an overall performance improvement of 2.02x. Figure 25 shows the results for running the base and optimized cases under the production environment. The base case results in a mean performance of 1025.9 seconds, and the mean performance of the optimized case is 499.2 seconds for a speedup of $2.06 \mathrm{x}$. The speedup achieved for the $95 \%$ confidence interval is between $2.00 \mathrm{x}$ and $2.10 \mathrm{x}$. The performance run in the low-variability environment accurately reflects the performance gain under the production environment. Selecting random samples from the production environment runs yields a speedup from between $1.80 \mathrm{x}$ to $2.31 \mathrm{x}$.

\section{CONCLUSIONS}

In this paper, we have characterized and quantified the different sources of variability on KNL-based Cray XC systems, and mitigation approaches were outlined wherever possible. We have identified the prominent sources of potential variability at the core, tile, node, and system levels. The variability observed at the core level may be primarily attributed to OS operations. This variability is most prominent when measurement intervals are short, on the order of milliseconds or less, and approaches zero for measurement intervals longer than a second. The impact on application performance, however, can still be significant as the over-burdened first core that runs OS processes can make synchronization calls expensive because of the resulting performance imbalance. Latencybound MPI collective operations can be affected by this noise. Core specialization is observed to help mitigate this noise and reduce the overall impact on the applications. 


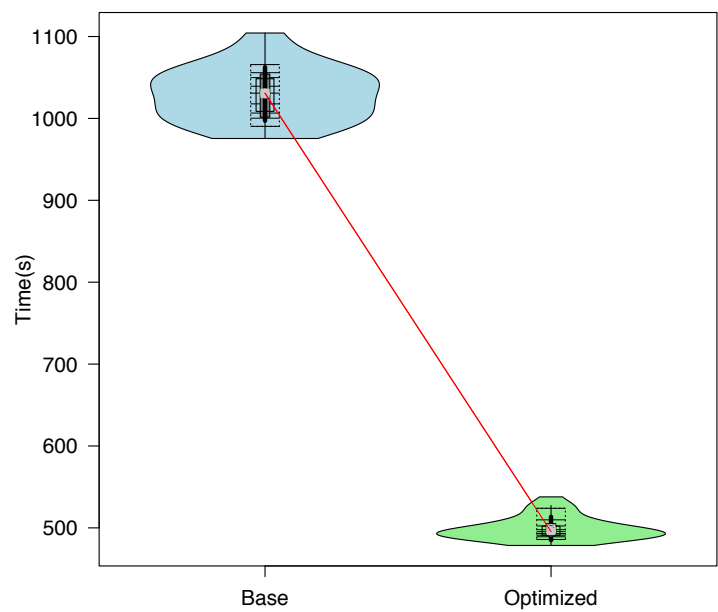

Figure 25: LAMMPS solve time comparison of the base case and the optimized case under the production environment

Another source of variability has been observed to occur at the tile level. The KNL uses a tile-based architecture with two cores on a tile sharing the L2 cache. The sharing of the caches by threads/processes can lead to cache contention with potential preferential treatment occurring between the two cores. This result manifests as a bimodal effect where performance differences occur between cores and produce around $20 \%$ core-to-core variability. While inefficient, using only one tile per core will eliminate variability from this source.

Another source of variability was found to occur at the node level when the KNL was utilized in cache mode. The direct-mapped MCDRAM cache was observed to contribute around 2X variability to the STREAM benchmark and around $20 \%$ variability to the Nekbone application. Performance variability was found to be reduced to less than $1 \%$ for bandwidth-intensive benchmarks, such as STREAM, when run in flat mode. An improved Zone sort algorithm and the use of huge pages are the other potential mitigations that remain to be explored.

The source of variability at the system level is the well-known issue of inter-job contention that occurs due to a shared network resource on the dragonfly topology. The level of variability on the Cray XC40 system was quantified using MPI benchmarks and real applications. For MPI collectives, performance was observed to vary by as high as 7X due to network contention and for the MILC application, a performance variability of around $70 \%$ from run to run was observed and attributable to variability in the communication performance.

Variability at any of the identified levels directly affects the performance of an application. The extent of the impact depends on the application characteristics such as being compute-bound, memorybound, or communication-bound. Hence, it is necessary to take variation into account when quantifying the performance of the applications. Experiments should be designed and reported with sufficient sample sizes to obtain statistically significant observations. The mitigations noted in this work can reduce the number of samples required and simplify the process of performance tuning.
Finally, given the characterization of the variability, performance tuning analysis can be carried out under a low-variability environment, which accurately predicts the expected performance improvement under the production environment without the need for gathering large sample sets to validate the statistical improvement.

\section{ACKNOWLEDGMENTS}

This research used resources of the Argonne Leadership Computing Facility, which is a DOE Office of Science User Facility supported under Contract DE-AC02-06CH11357. The authors would like to thank Brian Austin for sharing his insights on the cache mode variability and Sundaram Chintamani for discussions on the tile level variability.

\section{REFERENCES}

[1] Bilge Acun, Phil Miller, and Laxmikant V. Kale. 2016. Variation Among Processors Under Turbo Boost in HPC Systems. In Proceedings of the 2016 International Conference on Supercomputing (ICS '16). Article 6, 12 pages.

[2] Pete Beckman, Kamil Iskra, Kazutomo Yoshii, Susan Coghlan, and Aroon Nataraj. 2008. Benchmarking the Effects of Operating System Interference on Extremescale Parallel Machines. Cluster Computing 11, 1 (March 2008), 3-16.

[3] A. Bhatele, N. Jain, Y. Livnat, V. Pascucci, and P. T. Bremer. 2016. Analyzing Network Health and Congestion in Dragonfly-Based Supercomputers. In 2016 IEEE International Parallel and Distributed Processing Symposium (IPDPS). 93-102.

[4] Abhinav Bhatele, Kathryn Mohror, Steven H. Langer, and Katherine E. Isaacs. 2013. There Goes the Neighborhood: Performance Degradation Due to Nearby Jobs. In Proceedings of the International Conference on High Performance Computing, Networking, Storage and Analysis (SC '13). Article 41, 12 pages.

[5] Jiahao Chen and Jarrett Revels. 2016. Robust Benchmarking in Noisy Environments. In 20th Annual IEEE High Performance Extreme Computing Conference.

[6] CORAL. 2014. CORAL Application Benchmarks. (2014). https://asc.llnl.gov/ CORAL-benchmarks/

[7] Kurt B. Ferreira, Patrick Bridges, and Ron Brightwell. 2008. Characterizing Application Sensitivity to OS Interference Using Kernel-level Noise Injection. In Proceedings of the 2008 ACM/IEEE Conference on Supercomputing (SC '08). Article 19,12 pages.

[8] Ryan E. Grant, Kevin T. Pedretti, and Ann Gentile. 2015. Overtime: A Tool for Analyzing Performance Variation Due to Network Interference. In Proceedings of the 3rd Workshop on Exascale MPI (ExaMPI '15). Article 4, 10 pages.

[9] Alexander Heinecke, Greg Henry, Maxwell Hutchinson, and Hans Pabst. 2016. LIBXSMM: Accelerating Small Matrix Multiplications by Runtime Code Generation. In Proceedings of the International Conference for High Performance Computing, Networking, Storage and Analysis (SC '16). Article 84, 11 pages.

[10] Wim Heirman, Joni Dambre, Dirk Stroobandt, and Jan Van Campenhout. 2008. Runtime Variability in Scientific Parallel Applications. In Proceedings of the Fourth Workshop on Modeling, Benchmarking and Simulation at ISCA-35. 37-46.

[11] T. Hoefler and R. Belli. 2015. Scientific Benchmarking of Parallel Computing Systems. In Proceedings of the International Conference for High Performance Computing, Networking, Storage and Analysis (SC15). ACM, 73:1-73:12.

[12] Torsten Hoefler, Timo Schneider, and Andrew Lumsdaine. 2010. Characterizing the Influence of System Noise on Large-Scale Applications by Simulation. In Proceedings of the 2010 ACM/IEEE International Conference for High Performance Computing, Networking, Storage and Analysis (SC '10). 1-11.

[13] Intel. 2017. In Intel Xeon Phi Processor Performance Monitoring Reference Manual Volume 2: Events.

[14] Intel. 2017. In Intel Xeon Phi Processor Software - User Guide, Revision: 2.1. http://registrationcenter-download.intel.com/akdlm/irc_nas/11291/xppsl_ user_guide.pdf

[15] Nikhil Jain, Abhinav Bhatele, Xiang Ni, Todd Gamblin, and Laxmikant V. Kale. 2017. Partitioning Low-diameter Networks to Eliminate Inter-job Interference. In Proceedings of the IEEE International Parallel \& Distributed Processing Symposium (IPDPS '17).

[16] William Kramer and David Skinner. 2009. Consistent Application Performance at the Exascale. Int. F. High Perform. Comput. Appl. 23, 4 (Nov. 2009), 392-394.

[17] Edgar A. León, Ian Karlin, Abhinav Bhatele, Steven H. Langer, Chris Chambreau, Louis H. Howell, Trent D’Hooge, and Matthew L. Leininger. 2016. Characterizing Parallel Scientific Applications on Commodity Clusters: An Empirical Study of a Tapered Fat-tree. In Proceedings of the International Conference for High Performance Computing, Networking, Storage and Analysis (SC '16). Article 78, 12 pages.

[18] John D. McCalpin. 1995. Memory Bandwidth and Machine Balance in Current High Performance Computers. IEEE Computer Society Technical Committee on 
Computer Architecture (TCCA) Newsletter (Dec. 1995), 19-25.

[19] H. Sarp Oral, Feiyi Wang, David A. Dillow, Ross G. Miller, Galen M. Shipman, Don E. Maxwell, Jeffrey L. Becklehimer, Jeffrey M. Larkin, and David Henseler. 2010. Reducing Application Runtime Variability on Jaguar XT5. In CUG 10: Proceedings of Cray User's Group (CUG) Meeting.

[20] Allan Porterfield, Rob Fowler, Sridutt Bhalachandra, Barry Rountree, Diptorup Deb, and Rob Lewis. 2015. Application Runtime Variability and Power Optimization for Exascale Computers. In Proceedings of the 5th International Workshop on Runtime and Operating Systems for Supercomputers (ROSS '15). Article 3, 8 pages.

[21] Howard Pritchard, Duncan Roweth, David Henseler, and Paul Cassella. 2012 Leveraging the Cray Linux Environment Core Specialization Feature to Realize MPI Asynchronous Progress on Cray XE Systems. In Proceedings of the Cray User Group Conference.

[22] A. Sandberg, A. Sembrant, E. Hagersten, and D. Black-Schaffer. 2013. Modeling Performance Variation due to Cache Sharing. In 2013 IEEE 19th International Symposium on High Performance Computer Architecture (HPCA). 155-166.

[23] Joseph Schuchart, Daniel Hackenberg, Robert Schöne, Thomas Ilsche, Ramkumar Nagappan, and Michael K. Patterson. 2016. The Shift from Processor Power Consumption to Performance Variations: Fundamental Implications at Scale. Comput. Sci. 31, 4 (Nov. 2016), 197-205.

[24] D. Skinner and W. Kramer. 2005. Understanding the Causes of Performance Variability in HPC Workloads. In IEEE International. 2005 Proceedings of the IEEE Workload Characterization Symposium, 2005. 137-149.

[25] Avinash Sodani. 2015. Knights Landing (KNL): 2nd Generation Intel® Xeon Phi Processor. In Hot Chips 27 Symposium (HCS), 2015 IEEE. IEEE, 1-24.

[26] Brian Van Straalen, John Shalf, Terry Ligocki, Noel Keen, and Woo-Sun Yang. 2009. Scalability Challenges for Massively Parallel AMR Applications. In Proceedings of the 2009 IEEE International Symposium on Parallel\&Distributed Processing (IPDPS '09). 1-12.

[27] Nicholas J. Wright, Shava Smallen, Catherine Mills Olschanowsky, Jim Hayes, and Allan Snavely. 2009. Measuring and Understanding Variation in Benchmark Performance. In Proceedings of the 2009 DoD High Performance Computing Modernization Program Users Group Conference (HPCMP-UGC '09). 438-443.

[28] Xu Yang, John Jenkins, Misbah Mubarak, Robert B. Ross, and Zhiling Lan. 2016 Watch out for the Bully!: Job Interference Study on Dragonfly Network. In Proceedings of the International Conference for High Performance Computing, Networking, Storage and Analysis (SC '16). Article 64, 11 pages.

[29] W. Zhang, X. Ji, B. Song, S. Yu, H. Chen, T. Li, P. C. Yew, and W. Zhao. 2017 VarCatcher: A Framework for Tackling Performance Variability of Parallel Workloads on Multi-Core. IEEE Transactions on Parallel and Distributed Systems 28, 4 (2017), 1215-1228. 


\section{A ARTIFACT DESCRIPTION: RUN-TO-RUN VARIABILITY ON XEON PHI BASED CRAY XC SYSTEMS}

\section{A.1 Abstract}

This artifact contains the source code, input datasets and the build instructions that can be used to reproduce the variability experiments described and presented in the paper.

\section{A.2 Description}

A.2.1 Check-list (artifact meta information).

- Program: MILC, HACCmk, Nekbone, LAMMPS, MiniFE, FWQ, FTQ, Selfish, Reprompi, OSU Benchmarks, Statistical package for analysis

- Compilation: : CC (Cray wrapper around Intel compiler [intel 17.0.4.196])

- Run-time environment: Cray MPI (cray-mpich:7.6.0), Cray OS (SLES 12)

- Hardware: Argonne Theta system: Intel Xeon Phi 7230 and Cray Aries Interconnect with a Cray Cascade Dragonfly Topology

- Execution: see respective run scripts

- Output: Run time

- Experiment workflow: Download source code, compile source code, run the application, parse the output files and analyze the summary. See below for more detailed information.

- Publicly available?: Yes

A.2.2 How software can be obtained (if available). Most of the programs used are publicly available codes from the CORAL benchmark website at https://asc.llnl.gov/CORAL-benchmarks. However, all codes used in this paper are available at git site: Secure Git: https://xgitlab.cels.anl.gov/variability/sc17

A.2.3 Hardware dependencies. The experiments shown were performed on Argonne's Theta Supercomputer, Theta consists of Intel Xeon Phi 7230 nodes interconnected with a Cray Aries in a Cray Cascade dragonfly topology.

A.2.4 Software dependencies. All benchmarks were built and run on Argonne's Theta using Cray's cc compiler and other default parameters. Theta uses the Cobalt job scheduler. All software dependencies specific to each benchmark will be noted in a README file within each subdirectory.

A.2.5 Datasets. Several benchmarks use random nominal values; otherwise datasets are provided with each benchmark.

\section{A.3 Installation}

To access the code, clone the repository by running git clone https:/xgitlab.cels.anl.gov/variability/sc17

Each benchmark used in the paper has a seperate directory which will contain source code, build scripts, run scripts, and output parsing scripts (if required).

- Core Variability

- Selfish: OS Noise

- Matrix Multiplication: OS Noise
- FWQ: Timer

- HACCmk: Timer

- Matrix Multiplication: Timer

- Tile Variability

- HACCmk

- Matrix Multiplication

- Memory Variability

- STREAM

- System Variability

- OSU Benchmarks

- Applications

- MILC

- NEKBONE

- LAMMPS

As these experiments are primarily designed for Theta, the build commands provided give examples that should be adapted if they were to be used on other systems.

\section{A.4 Experiment Workflow}

Each benchmark will have a "./run.sh" script which details how to run the application on Argonne's Theta system. Additionally a README file will explain the various options of the run script and output.

For example:

cd./corevariability/matrix_multiplication

make clean; make all

./run.sh

\section{A.5 Evaluation and Expected Results}

For each benchmark, the metric used to calculate variability is the maximum to minimum difference between the runtime across several runs or several cores.

For example: The Selfish benchmark is used to identify OS noise, by running the selfish benchmark on each core and using MPI to aggregate the results across all the used cores.

Each application will have a script to extract the data from the $\log$ files created during the run. This data will automatically be created in the respective folder. 